\title{
Facteurs prédictifs de décès au cours de la TB pulmonaire chez les PvVIH au CNHU Fann de Dakar
}

\author{
Harouna Amadou M.L.
}

Service de Maladies infectieuses, Hôpital Régional de Maradi, Faculté des

Sciences de la Santé, Université de Maradi, Niger

\section{Mahamadou Doutchi}

Service de Maladies infectieuses et Tropicales, Hôpital National de Zinder,

Faculté des Sciences de la Santé, Université de Zinder, Niger

Ousmane Abdoulaye

Laboratoire CHR de Maradi, Université de Maradi, Niger

Amadou $O$.

Hôpital Regional de Maradi, Faculté des Sciences de la Santé, Université de Maradi, Niger

Abdou Neino $M$.

Kadri Sani

Hôpital Regional de Maradi, service de Médecine Interne

Abdoul Aziz Garba

Service de Médecine interne, Hôpital National de Zinder-Niger

CT Ndour

Service de Maladies infectieuses et Tropicales, CNHU Fann,

Faculté des Sciences de la Santé, Université de Dakar

Doi: 10.19044/esj.2018.v14n36p1 URL:http://dx.doi.org/10.19044/esj.2018.v14n36p1

Abstract

Objectives: In sub-Saharan Africa, tuberculosis is the first pulmonary-located opportunistic infection and the leading cause of death among people living with HIV (PLWHA). This paper focuses on studying the predictive factors of death in people co-infected with pulmonary tuberculosis and HIV at the Ibrahima DIOP MAR clinic for infectious and tropical diseases at the CHNU Fann in Dakar. Materials and Methods: This was a retrospective, descriptive, and analytical study conducted from 1st January 1998 to 31st December 2009. It has been of interest to all patients with a successful pulmonary tuberculosis diagnosis and positive HIV status. Epidemiological, clinical, para-clinical, and progressive parameters were studied. Results: A total of 665 cases of tuberculosis/HIV co-infection were observed during the study period, of which 207 deaths or lethality (31.37\%) 
was recorded. The average age of patients was $39 \pm 9$ years with extremes of 5 and 74 years. The average length of hospitalization was $31.67 \pm 20.67$ with extremes of 4 and 312 days for surviving patients compared to $19.00 \pm 16.42$ with extremes of 1 to 90 days for deceased patients. Immunosuppression was significantly associated with death $(\mathrm{p}=0.001)$ with a median CD4 of 32.50 elts/mm3. Lethality was significantly elevated in the presence of dyspnea $(\mathrm{p}=$ 0 , 00014), consciousness disorder $\left(\mathrm{p}=10^{-6}\right)$, motor deficit $(\mathrm{p}=0.008)$, and hemoptysis $(\mathrm{p}=0.03)$. Conclusion: Tuberculosis/HIV co-infection is a common association in Africa. Here, we are talking about a deadly duo. Taking it requires a good knowledge of the factors of poor prognosis and a good integration of the two programs.

Keywords: Lethality, co-infection, pulmonary tuberculosis, HIV, Dakar

\section{Resume}

En Afrique sub-saharienne, la tuberculose constitue la première infection opportuniste à localisation pulmonaire et la première cause de décès chez les personnes vivant avec le VIH (PvVIH). L'objectif de ce travail est d'étudier les facteurs prédictifs de décès chez les personnes Co-infectées par la tuberculose pulmonaire et le VIH à la clinique des maladies infectieuses et tropicale Ibrahima DIOP MAR du CHNU Fann de Dakar. Matériels et méthodes : Il s'agissait d'une étude rétrospective, descriptive et analytique réalisée du $1^{\mathrm{er}}$ janvier 1998 au 31 décembre 2009. Elle a intéressé tous les patients dont le diagnostic de tuberculose pulmonaire a été retenu et ayant une sérologie VIH positive. Les paramètres épidémiologiques, clinique, para clinique et évolutifs ont été étudiés. Résultats : Au total 665 cas de coinfections tuberculose $/ \mathrm{VIH}$ ont été observés pendant la période d'étude dont 207 décès soit une létalité : de 31,37\%.L'âge moyen des patients était de $39 \pm 9$ ans avec des extrêmes de 5 et 74 ans. La durée d'hospitalisation moyenne a été de 31,67 $\pm 20,67$ avec des extrêmes de 4 et 312 jours pour les patients survivants contre 19,00 $\pm 16,42$ avec des extrêmes de 1 à 90 jours pour les patients décédés. L'immunodépression a été significativement associée au décès $(\mathrm{p}=0,001)$ avec une médiane de CD4 de 32,50 élts $/ \mathrm{mm}^{3}$. La létalité a été significativement élevée en présence de dyspnée $(\mathrm{p}=0,00014)$, de trouble de la conscience $\left(p=10^{-6}\right)$ de déficit moteur $(p=0,008)$ et d'hémoptysie $(p=0,03)$. Conclusion : La coïnfection tuberculose/ VIH est une association fréquente en Afrique. On parle de duo mortel. La prise passe par une bonne connaissance des facteurs de mauvais pronostique et nécessite une bonne intégration des deux programmes.

Mots-clés: Létalité, coïnfection, tuberculose pulmonaire, VIH, Dakar 


\section{Introduction}

La tuberculose constitue un problème majeur de santé publique au niveau mondial. Selon les estimations de l'OMS de 2012, il y a environ 8,7 millions de nouveaux cas de tuberculose à l'échelle mondiale, dont $13 \%$ chez les personnes vivant avec le VIH (WHO, 2012). En 2011 la tuberculose a été responsable de 1,4 million de décès, dont 430000 séropositifs (WHO, 2012). Elle est la 2e cause de mortalité par maladie infectieuse après l'infection a VIH dans le monde. Un tiers de la population mondiale est infecté par Mycobacterium tuberculosis (Boulahbal \& Chaulet, 2004 ; WHO, 2012). La tuberculose a connu une nette recrudescence dans le monde et principalement en Afrique subsaharienne depuis l'avènement du VIH dans les années 80 (Harries, Zachariah \& Lawn, 2009). Ainsi la co-infection tuberculose/ VIH (TB/VIH) constitue une association péjorative ce qui amène certains auteurs à parler de duo diabolique (Laureilllard, 2007). La TB est toujours la première cause d'infection opportuniste et de décès chez les PVVIH en Afrique subsaharienne (Statistiques mondial sur le VIH ONUSIDA, 2017; Dagnra et al., 2011). L'objectif de ce travail est d'étudier les facteurs prédictifs de décès chez les personnes coinfectées par la tuberculose pulmonaire et le VIH à la clinique des maladies infectieuses et tropicales Ibrahima DIOP MAR du CHNU Fann de Dakar.

\section{Patients et Methodes \\ Type d'étude}

Il s'agissait d'une étude descriptive rétrospective réalisée à partir de dossiers de patients dont le diagnostic de tuberculose pulmonaire a été retenu et ayant une sérologie VIH positive.

\section{Population d'étude}

L'étude a intéressé les patients suivis dans le service des maladies infectieuses et tropicales du CHNU Fann Dakar (Sénégal), sur la période 1998 à 2009. Ont été' exclus tous les patients co-infectés TB/VIH réfères dans d'autres structures sanitaires pour leur prise en charge et tous les patients TB/VIH déjà sous traitement antituberculeux ou antirétroviral avant janvier 1998.

\section{Définitions de cas}

Le diagnostic de tuberculose a été retenu sur la présence de bacilles acido-alcoolo résistants (BAAR) dans les produits pathologiques après coloration de Ziehl-Nielsen et en l'absence de confirmation bactériologique sur des arguments épidémiologiques (notion de contage, absence de 
vaccination, terrain),cliniques, radiologiques, histologiques (mise en évidence du granulome tuberculoïde par examen anatomopathologique des pièces biopsiques) et thérapeutiques (amélioration du tableau clinique sous traitement après échec d'un traitement antibiotique non spécifique), la culture n'étant pas souvent effectuée du fait de la pauvreté du plateau technique. La dénutrition était définie comme un indice de masse corporel (IMC) inférieur à 18,5kg/m2 .

\section{Variables étudiées et analyse statistiques}

A partir d'un questionnaire standard, nous avons recueilli les données socio démographiques, épidémiologiques, cliniques, para cliniques, thérapeutiques et évolutives.

La saisie a été faite grâce au logiciel Epi data, puis les données ont été exportées vers Excel et analysées grâce au logiciel SPSS. Les proportions et les caractéristiques des patients décédés ont été comparé aux patients guéris. Le test de Kruskas Walis a été utilisé pour rechercher l'association entre les variables quantitatives et la mortalité, alors les tests de $K H I^{2}$ et Fischer étaient utilisés pour le lien entre les variables qualitatives et la mortalité. La valeur de p<0,05 a été considérée comme statistiquement significative.

\section{Prise en charge des patients}

Tous les patients ont bénéficié d'une prise en charge thérapeutique conformément aux recommandations du Programme national de lutte contre la tuberculose et aux recommandations de l'OMS en vigueur sur la prise en charge de l'infection à VIH adaptées au contexte de l'initiative sénégalaise d'accès aux antirétroviraux (ISAARV).

\section{Resultats}

\section{Aspects épidémiologiques}

Durant la période d'étude, 26318 patientes toutes pathologies confondues ont été hospitalisés au CNHU Fann dont 1194 cas de tuberculose soit 4,54\%. Au total 665 cas de tuberculose pulmonaire chez des patients à sérologie VIH positive ont été colligés. Une prédominance masculine a été notée avec un sex-ratio de 1,35. La moyenne d'âge de nos patents a été de 39,5 $\pm 9,5$ ans avec des extrêmes de 5 et 74 ans. La moyenne d'âge a été plus élevée chez les patients décédés (40,58 ans $\pm 11,09$ contre 39,23 ans $\pm 9,36)$ sans différence statistiquement significative $(\mathrm{P}=0,2)$.

\section{Aspects cliniques}

La majorité des patients $(93,23 \%)$ présentait un profil VIH-1 et 18,34 $\%$ avaient des antécédents de tuberculose. Les comorbidités retrouvées étaient le diabète, l'hypertension artérielle et la paralysie faciale. Un patient sur quatre avaient des antécédents d'infections opportunistes à type de candidose bucco- 
œsophagienne, de diarrhée chronique, de prurigo ou de zona. Des proportions de fièvre $(97,6 \%)$, amaigrissement $(71 \%)$, toux chronique $(83,75 \%)$, adénopathies périphériques $(58 \%)$, hémoptysie $(23,6 \%)$ et dyspnée $(4,6 \%)$ ont été retrouvées dans notre série.

\section{Aspects para cliniques}

Dans notre série, Le taux moyen d'hémoglobine était de 7,37g/dl chez les patients survivants contre 7,28g/dl chez les décédés (tableau 1). Le taux moyen de lymphocytes T CD4+ a été plus élevé chez les patients guéris, $118,82 \pm 16,9$ contre $66,79 \pm 8,19$ pour les patients décédés. La médiane des CD4 a été respectivement de 66 et 32,5 éléments/mm3 pour les patients survivants et décédés. Une anémie a été retrouvée chez 44 patients $(66,8 \%)$ avec un taux d'hémoglobine moyen à 7,59 $\pm 1,8 \mathrm{~g} / \mathrm{dl}$. Nous avons trouvé une VS (vitesse de sédimentation) accélérée à la première heure avec une valeur moyenne de 82,54 $\pm 79 \mathrm{chez}$ les patients guéris contre 76,73 $\pm 19 \mathrm{chez}$ les patients décédés (Tableau 1).

\section{Aspects thérapeutiques et évolutifs}

Sur le plan thérapeutique, la chimio prophylaxie au cotrimoxazole a été instaurée chez 540 patients $(82,44 \%)$. Au plan évolutif, nous avions enregistré 207 décès soit un taux de létalité de 31,37\%. La létalité a été importante chez les patients qui ont eu une durée moyenne d'hospitalisation plus courte $(19,00 \pm 16,42$ versus $31,67 \pm 20,67),\left(p=10^{-6}\right)$ (Tableau 1). La létalité a été significativement associée à la présence de dyspnée $(\mathrm{p}=0,00014)$, de trouble de la conscience $\left(p=10^{-6}\right)$, de déficit moteur $(p=0,008)$ et d'hémoptysie $(p=0,03)$ (Tableau 1). L'anémie sévère avec un taux d'hémoglobine inférieur à $7 \mathrm{~g} / \mathrm{d}$ l ont été associés de manière significative au décès $(p=0,04)$.Un taux d'hémoglobine entre 9 et $13 \mathrm{~g} / \mathrm{dl}$ confère une protection de $77 \%$ contre le décès comparé à une valeur de référence $(<7 \mathrm{~g} / \mathrm{dl})$. L'immunodépression a été fortement associée au décès $(p=0,001)$. La médiane de 32,50 éléments $/ \mathrm{mm}^{3}$ de $\mathrm{CD}_{4}$ chez les patients décédés. Nous avons retrouvé une association significative entre une VS accélérée et la mortalité $(\mathrm{p}=0,002)$ (Tableau 1).

\begin{tabular}{|c|c|c|c|c|}
\hline \multicolumn{7}{|c|}{ Tableau 1. Facteurs prédictifs de décès } \\
\hline Paramètres & Survivants & Décédé & $*$ ORIC95\% & p \\
\hline Homme & 242 & 130 & $1,44[1,02-2,01]$ & 0,034 \\
\hline Femme & 206 & 77 & - & \\
\hline Moyenne d'âge & $39,23 \pm 9,36$ & $40,58 \pm 11,9$ & - & - \\
\hline Dyspnée & 221 & 135 & $0,51[0,36-0,72]$ & 0,00014 \\
\hline Adénopathies & 80 & 25 & $1,54[0,95-2,5]$ & 0,07 \\
\hline Fièvre & 443 & 201 & $0,8[0,33-2,30]$ & 0,73 \\
\hline Hémoptysie & 22 & 19 & $0,49[0,26-0,94]$ & 0,029 \\
\hline
\end{tabular}




\begin{tabular}{|c|c|c|c|c|}
\hline Troubles de la conscience & 33 & 39 & $0,33[0,20-0,54]$ & 0,0000 \\
\hline Déficit moteur & 13 & 15 & $0,37[0,17-0,80]$ & 0,0087 \\
\hline Vitesse de sédimentation & $82,54 \pm 79$ & $76,73 \pm 19$ & & 0,002 \\
\hline Moyenne Hb & 7,28 & 7,37 & & - \\
\hline Médiane CD4 & 66 & 32,5 & & 0,001 \\
\hline Durée d'hospitalisation & $31,66 \pm 20$ & $19,00 \pm 16$ & - & 0,000001 \\
\hline
\end{tabular}

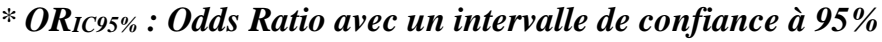

\section{Discussion}

\section{Sur le plan épidémiologique}

La Tuberculose est une maladie infectieuse contagieuse provoquée le Mycobactérium tuberculosis. Une petite proportion (5-15\%) des 1.7 milliard de personnes contaminées développe la maladie. Le VIH est à la fois un facteur de risque d'infection à mycobacterium tuberculosis et un facteur déterminant d'évolution vers la tuberculose maladie (WHO, 2017).

$\mathrm{Au}$ cours de cette étude, 655 cas de coïnfection TB-VIH ont été retrouvés sur un total de 1194 suivit pour tuberculose soit une séroprévalence globale de 54,8\%. Cette séroprévalence est nettement supérieure celle rapportée au Togo par A.Y. Dagnra (23,7 \%) (Dagnra et al., 2011). Certaines auteurs ont rapportés des chiffres pouvant aller jusqu'à $75 \%$ (Ondounda et al. 2011; WHO, 2009).

Dans notre étude, le sexe masculin prédominait chez les patients coinfectés avec une différence significative. Cette prédominance masculine chez les tuberculeux, est décrite dans la littérature comme le confirme les études de Mbena au Mali et Grant en Côte d'Ivoire avec respectivement $53 \%$ et $62 \%$ (Mbe'na, 2015; Grant et al. 1999). Ailleurs au Brésil et en Indonésie, Santos et Fredy avaient constaté les mêmes résultats avec respectivement $76 \%$ et 80 $\%$ (Santos et al. 2011; Fredy et al., 2011). Par contre, une prédominance féminine a été notée par Breton en République centrafricaine et Mugisha en Ouganda (52\% contre $36 \%$; < 0,05) (Breton et al., 2002; Mugisha et al., 2006). L'âge médian a été de 40,5 ans. Ce résultat est comparable à celui de Fortes (Fortes et al., 2011) à Dakar, Oudounda au Gabon (Ondounda et al., 2012) Grant en Côte d'Ivoire avec respectivement des âges médian de 40 ans, 40 ans et 39 ans. En Afrique du Sud et en Ouganda, pays à forte incidence de tuberculose, la moyenne d'âge été inférieure à celle retrouvée dans notre série, elle était de 32 ans (Badri et al., 2001; Harries et al.).

\section{Au plan clinique}

Des proportions de fièvre $(97,6 \%)$, amaigrissement $(71 \%)$, toux chronique $(83,75 \%)$, adénopathies périphériques (58\%), hémoptysie $(23,6 \%)$ et dyspnée $(4,6 \%)$ ont été retrouvées dans notre série. Sylla a rapporté dans sa série des proportions de toux chronique de dyspnée et 
d'hémoptysie de respectivement 59\%, 4,6\% et 23,6\% (Sylla et al., 2017). Nous avons retrouvé des signes neurologiques à type de trouble de la conscience et de déficit moteur dans respectivement $10,87 \%$ et $4,2 \%$.

La létalité a été significativement élevée en présence de dyspnée ( $\mathrm{p}=$ $0,00014)$, de trouble de la conscience $\left(\mathrm{p}=0,10^{-6}\right)$ de déficit moteur $(\mathrm{p}=0,0087)$ et d'hémoptysie $(\mathrm{p}=0,03)$ (Tableau 1).

\section{Au plan para clinique}

Le taux moyen d'hémoglobine était de $7,37 \mathrm{~g} / \mathrm{dl}$ chez les patients survivants contre 7,28g/dl chez les décédés (Tableau 1). Des taux moindres ont été retrouvés par Ka et Senghor (Leeds et al., 2012; Ka et al., 2016). L'anémie dans la coïnfection TB/VIH peut être inflammatoire, nutritionnelle ou centrale par cytopathie virale (Coyle, 1997; Girard et al., 2007). Dans notre série l'anémie peut se justifier par le faible niveau économique de notre population d'étude. La tuberculose et l'infection à VIH ont un effet délétère sur l'hématopoïèse et le système immunitaire. L'analyse multi variée montre qu'un taux d'hémoglobine entre 9 et $13 \mathrm{~g} / \mathrm{dl}$ confère une protection de $77 \%$ contre le risque de décès $(\mathrm{p}=0,04)$ par rapport à un taux moyen d'hémoglobine $(<7 \mathrm{~g} / \mathrm{dl})$. Nous avons trouvé une VS (vitesse de sédimentation) accélérée à la première heure avec une valeur moyenne de 82,54 \pm 79 chez les patients guéris contre $76,73 \pm 19$ chez les patients décédés (Tableau 1). Une VS accélérée a été significativement associée à la mortalité $(\mathrm{p}=0,002)$.Plusieurs auteurs ont commenté cette association (Ait Khaled \& Enarson, 1999; Pilly, 2008). L'accélération de la VS chez les patients VIH positifs serait la conséquence de l'anémie chronique fréquemment observée chez ces derniers (Clark et al., 2002).

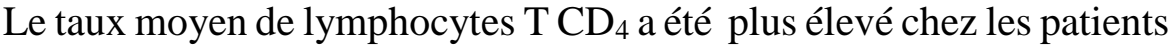
non décédés, 118,82 contre 66,79 pour les patients décédés. La médiane des CD4 a été respectivement de 66 et 32,5 éléments $/ \mathrm{mm}^{3}$ pour les patients survivants et décédés (Tableau 1). L'immunodépression a été significativement associée au décès $(\mathrm{p}=0,001)$.Cette immunodépression sévère (CD4 <350) a été décrite dans la littérature comme le confirme les études de Fortes à Dakar (86 \%), Oudounda au Gabon 177/mm3 et Grant en Cote d'ivoire, 167/mm3 (Grant et al., 1999; Fortes et al., 2011; Ondounda et al., 2012). Dans la série de A.Sylla en côte d'ivoire, la médiane des CD4 a été de 94 éléments $/ \mathrm{mm}^{3}$ (Sylla et al., 2017). Bemba au Congo Brazaville a

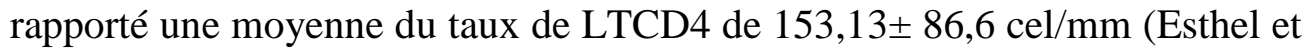
al., 2016). Ces résultats traduisent un état d'immunodépression sévère chez les patients co-infectés TB/VIH conséquence du retard diagnostic et thérapeutique dans les pays à faible revenu. Cet état de fait entretient cette spirale mortelle en ce sens que l'infection à VIH favorise la réactivation d'une infection latente à $\mathrm{M}$. tuberculosis vers une TB maladie et $\mathrm{M}$. tuberculosis 
favorise la réplication du VIH accélérant ainsi l'évolution naturelle de l'infection vers l'immunodépression (Dagnra et al. 2011).

\section{Au plan thérapeutique et évolutif}

Dans notre étude $82,44 \%$ de nos patients étaient sous chimioprophylaxie au cotrimoxazole. Au Benin et en Ethiopie, des taux respectifs de $99 \%$ et $92 \%$ ont été retrouvés (Assefa et al., 2011; Agadokpessi et al., 2012). Ces taux témoignent de la bonne application des recommandations de l'OMS sur la chimioprophylaxie au cotrimoxazole quand le taux de CD4 est inférieur à 350 cellules/mm3. La mortalité liée à la tuberculose est particulièrement élevée chez les patient VIH (S.pontier, 2008). Dans notre série nous avons enregistrés 207 cas de décès soit une létalité hospitalière de $31,37 \%$ (Tableau 1 ), comparable au résultat d'A. Sylla (31,1\%) (Sylla et al., 2017). Nous avons trouvé une différence significative de létalité selon les sexes $(\mathrm{P}=0,0034)$.

La durée moyenne d'hospitalisation a été de $31,66 \pm 20$ jours pour les survivants avec des extrêmes de 4 à 312 jour contre 19,00 \pm 16 jours pour les décédés avec des extrêmes de 1 à 90 jours. Une durée d'hospitalisation longue a été un facteur de bon pronostic dans notre étude. $\left(\mathrm{P}=10^{-6}\right)$ (Tableau 1$)$.

\section{Conclusion}

La coïnfection tuberculose/ VIH est une association fréquente en Afrique. On parle de duo mortel. La prise en charge passe par une bonne connaissance des facteurs de mauvais pronostique et nécessite une bonne intégration des deux programmes.

\section{Conflit D'intérêts}

Aucun.

\section{Remerciements}

A Tout Le Personnel Du Chhu Fann De Dakar, Au Ministère De La Santé Publique Du Sénégal.

\section{Contribution Des Auteurs}

Tous Les Auteurs Ont Lu Et Approuvé La Version Finale De Ce Manuscrit.

\section{References:}

1. Agadokpessi, G., Ade, G., Ade, S. et al. (2012). Management of tuberculosis and HIV co-infection in Cotonou, Be'nin. Med Mal Infect; 42 : 561-6. 
2. Ait Khaled, N. \& Enarson, D. (1999). Tuberculose: Manuel pour les étudiants en médecine OMS-Union international contre la tuberculose et les maladies respiratoires, 1999: 149 pages.

3. Assefa, D., Melaku, Z., Gadissa, T., Negash, A., \& Hinderaker, SG. (2011). Dépistage intensifie' des cas de tuberculose chez des sujets infecte's par le virus de l'immunode'ficience humaine dans une clinique hospitalie' re enE' thiopie. Int J Tuberc Lung Dis; 15 : 411-3.

4. Badri, M., Ehrlich, R., Wood, R., Pulerwitz, T., Maartens, G. (2001). Association between tuberculosis and HIV disease progression in high tuberculosis prevalence area. Int J Tuberc Lung Dis; 5 : 225-32.

5. Boulahbal, F. \& Chaulet, P. (2004). La tuberculose en Afrique, e'pide'miologie et mesures de lutte. Med Trop; 64 : 224-8.

6. Breton, G., Service, YB., Kassa-Kelembho, E., Mbolidi, CD., Minssart, P. (2002). Tuberculose et VIH a' Bangui. Re'publique centrafricaine : forte pre'valence et difficultés de prise en charge. Med Trop; $62:$ 623-6.

7. Clark, T.D., Mmiro, F., Ndugwa, C., et al. (2002). Risk factors and cumulative incidence of anaemia among human immunodeficiency virus-infected children in Uganda. Ann Trop Paediatr; 22: 11-7.

8. Coyle, T.E. (1997). Hematologic complications of human immunodeficiency virus infection and the acquired immunodeficiency syndrome. Med Clin North Am; 81: 449-470

9. Esthel Lee Presley Bemba, Régis Gothard Bopaka, Régis Moyikoua, Rolland Ossibi-Ibara, Norela Bibiane Ebenga-Somboko, Syn Nerval Toungou, Paunel God'hervé Matondot, Boris Kevin Ossale-Abacka, Franck Hardain Okemba-Okombi, Joseph Mboussa (2016). Tuberculose pulmonaire à Brazzaville en hospitalisation pneumologique: impact du diagnostic tardif à l'infection au VIH sur les anomalies radiographiques Pan African Medical Journal; 24:259 doi:10.11604/pamj.2016.24.259.9456

10. Fortes, LD., Manga, NM., Diop, SA., et al. (2011). Profil actuel de patients infectés par le VIH hospitalisés à Dakar (Sénégal). Bull Soc Path Exot; 104 : 366-70.

11. Fredy, FC., Liwang, F., Kurniawan, R., Nasir, AU. (2012). The correlation between CD4+ T-lymphocyte count and tuberculosis form in TB-HIV co-infected patients in Indonesia. Acta Med Indones; 44 : 122-7.

12. Girard, P.M., Pialoux, G., Katlama, C. VIH (2007). editor: DOIN; 2007.

13. Grant, AD., Sidibe', K., Domoua, K. et al. (1998). E'ventail des maladies chez les adultes infecte's par le VIH hospitalise's dans une 
unite' de médecine respiratoire a' Abidjan, Co`te d'Ivoire. Int J Tuberc Lung Dis; 2 : 926-34.

14. Harries, AD., Zachariah, R., \& Lawn, SD. (2009). Les soins du VIH chez les patients coinfectés par la tuberculose: une perspective provenant d'Afrique subsaharienne. Int J Tuberc Lung Dis; 13 : 6-16.

15. Ka, D., Diop Nyafouna, SA., Ndour, CT., et al. (2016). Tuberculose multifocale : aspectsépidémiologiques, cliniques, para cliniques et évolutifs selon le statut sérologique VIH à la clinique des maladies infectieuses du CHNU de Fann de Dakar. Rev. Cames Sante’; 4 : 727.

16. Laureilllard, D. (2007). Tuberculose chez le patient infecte' par le VIH. Se'minaire Tuberculose IFMT-MS Mai Cambodge.

17. Leeds, IL., Magee, MJ., Kurbatova, EV. et al. (2012). Site of extrapulmonary tuberculosis is associated with HIV infection. Clin Infect Dis; 55 : 75-81.

18. Mbe'na, CT. (2015). Aspects e'pide'miologiques, clinique et the'rapeutique de la co-infection VIH/tuberculose dans les centres de sante' de re'fe'rence des communes I, II et III du district de Bamako. [The'se de me'decine, Universite' de Bamako]. Mali, keneya.net/fmpos/theses/2010/med/pdf/10M359.pdf (consultation 29 aou^t 2015).

19. Mugisha, B., Bock, N., Mermin, J. et al. (2006). De'pistage et traitement pre'ventif de la tuberculose dans un centre volontaire pour le VIH en Ouganda. Int J Tuberc Lung Dis; 10 : 761-7.

20. Ondounda, M., Ilozue, C., Mounguengui, D., Magne, C., Nzenze, JR. (2011). Aspects cliniques et radiologiques de la tuberculose au cours de l'infection par le VIH à Libreville, Gabon. Med Trop 2011 ; 71 : 253-256.

21. Ondounda, M., Magne, C., Mounguengui, D. et al. (2012). Morbidity and mortality in HIV - infected patients in the Military Hospital in Libreville (Gabon). MedSante' Trop; 22 : 334-5.

22. Pilly, E. (2008). Maladies infectieuses et tropicales CMIT, Traitement antituberculeux, In E.Pilly : vivactis Plus Ed.

23. Santos Neto, M., Da Silva, FL., De Sousa, KR. et al. (2012). Clinical and epidemiological profile and prevalence of tuberculosis/HIV coinfection in a regional health district in the state of Maranhao, Brésil. J Bras Pneumol; 38 : 724-32.

24. Statistiques mondial sur le VIH ONUSIDA, 2017

25. Sylla, A., Marchou, B., Kassi, N., Ello, N., Aba, T., Kouakou, G., Moussou, C., Ehui, E., Eholié, S., Biassagnéné, E. (2017). Coinfection tuberculose/VIH : à propos de 717 cas suivis dans un service de maladies infectieuses en Afrique subsaharienne. Médecine et 
Maladies Infectieuses Volume 47, Issue 4, supplement, June, Pages S137- S138.

26. S. pontier : le poumon du sujet infecté par le VIH. Rev Mal Respir $2008 ; 25: 53-57$

27. Tidjani, O. (2011). Prévalence de la co-infection VIH-tuberculose et impact de l'infection à VIH sur l'évolution de la tuberculose pulmonaire au Togo Bull. Soc. Pathol. Exot. 104:342-346.

28. WHO (2009). Global tuberculosis control: a short update to the 2009 report WHO/HTM/TB/2009.4262009.

29. WHO (2012). Rapport OnuSida sur l'e'pide'mie mondiale de sida, 2012. Gene've :OnuSida, 2012.unaids.org/sites/default/files/media_asset/20121120_UNAIDS_ Global_Report_2012_with_annexes_fr_1.pdf (consultation 29 août 2015). Harries AD, Zachariah R, Lawn SD. Les soins du VIH chez les patients coinfectés par la tuberculose : une perspective provenant d'Afrique subsaharienne.Int J Tuberc Lung Dis 2009 ; 13 : 6-16.

30. WHO (2017). TB/HIV Fact Sheet. Global Tuberculosis Report 2017. Accessed from http://www.who.int/entity/tb/publications/ global_report/en/index.html Cited 15.12.17. 